\title{
23. Mißt der Aberrationswinkel im Fall einer Dispersion des Äthers die Wellengeschwindigkeit? $\left.{ }^{1}\right)$ von $P a u l E h r e n f e s t$.
}

In den Lehrbüchern der Optik wird gelegentlich die Frage berührt, ob der reine Äther eine merkliche Dispersion aufweist. Daran knüpft sich etwa folgende Erörterung ${ }^{2}$ ): Die Römersche Lichtgeschwindigkeitsmessung operiert mit der Fortpflanzungsgeschwindigkeit abgeschnittener Wellenzüge; dementsprechend liefere sie die „Gruppen"-Geschwindigkeit $U$; die Messung des Aberrationswinkels liefere hingegen direkt die Wellengeschwindigkeit $V$. Insoweit die beiden so erhaltenen Resultate übereinstimmen, dürfe man schlieBen, daB das Licht im Interplanetarraum sich dispersionsfrei fortpflanzt.

Die Behauptung, daß der Aberrationswinkel die Wellengeschwindigkeit miBt, scheint zum erstenmal von Lord Rayleigh ${ }^{3}$ ) aufgestellt worden zu sein. An der betreffenden Stelle setzt Lord Rayleigh auseinander (anläBlich der Experimente von Young und Forbes, 1881), daB die Fizeausche, Foucaultsche und Römersche Methode mit abgeschnittenen Wellenzïgen operieren und deshalb die Gruppengeschwindigkeit $U$ liefern. Bezüglich der Messung des Abberrationswinkels hingegen sagt er: "The former does not depend upon observing the propagation of a peculiarity impressed upon a train of waves, and therefore has no relation to $U$. If we accept the usual theory of aberration as satisfactory the result of comparison between the coefficient found by observation and the solar parallax is $V$-the wave velocity."

Durch die folgende Schematisation der Aberrationsmessung möchte ich ersichtlich machen, dab auch sie beruht ,upon

1) Aus einem im Herbst 1908 vor der Petersburger physikalischen Gesellschaft gehaltenen Vortrag.

2) R. Wood p. 18; P. Drude p. 116; O. D. Chwolson II, p. 246.

3) Lord Rayleigh, Scientif. Papers I. p. 587. 1881. 
observing the propagation of a peculiarity impressed upon a train of waves". - Die Figur stellt zwei parallele unendliche Platten dar, jede mit einer Öffnung versehen und mit gemeinsamer konstanter Geschwindigkeit in Richtung ihrer Erstreckung nach rechts laufend. Auf die obere Ebene mögen normal monochromatische Lichtstrahlen auffallen. Wir bestimmen den Winkel, um wel-

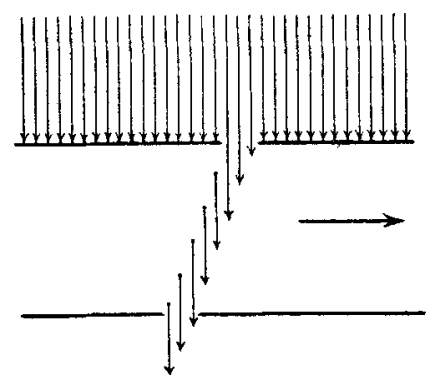
chen die untere Öffnung gegen die obere verschoben sein muB, damit der unter der unteren Öffnung befindliche (mitbewegte) Beobachter möglichst viel Licht erhalte. Benutzt man den Begriff „Lichtstrahl", so erkennt man ohne weiteres, daß jener Winkel sich durch die Geschwindigkeit bestimmt,

mit der die abgeschnittenen Lichtstrahlstücke abwärts wandern. Besitzt der Äther Dispersion, so liefert also auch die Messung des Aberrationswinkels nicht die Wellengeschwindigkeit $V$.

Versucht man zur Vermeidung des miBlichen Begriffes „Lichtstrahl" mit den Wellenebenen zu operieren, so erhält man folgendes Bild: die obere Ebene stanzt - wenn man dem Sinne der Überlegung gemäb von Beugung absieht aus jeder Wellenebene ein Kreisscheibchen heraus und die Kreisscheibchen wandern - jede gegen ihren $\nabla$ orgänger verschoben - nach abwärts. Achtet man nur auf die mittlere Partie einer solchen Wellenscheibe, so wandert sie natürlich mit Wellengeschwindigkeit, und diese scheint also - im Sinne Rayleighs - zu bestimmen, um welchen Winkel die untere Öffnung verspätet sein muB, damit die Wellenscheiben möglichst vollständig durch sie durchschlüpfen können. Man darf aber nicht vergessen, daß die Dispersion den (im Sinne der Erdbewegung) vorderen und hinteren Rand jeder Wellenscheibe während der Abwärtswanderung in schwer zu übersehender Weise nach und nach verändert und dadurch die günstigste Lage der unteren Öffnung beeinfluBt.

Die folgende Schematisation der Fizeauschen Zahnradmethode soll noch zeigen, dab die Messung der Lichtgeschwindig- 
keit mit Hilfe der Aberration und durch die Fizea usche Zahnradmethode für die hier behandelte Frage prinzipiell identisch sind: Zwei einander parallele Kreisscheiben, jede nahe der Peripherie durchlocht, seien durch eine lange rasch rotierende Achse verbunden. Auf die rechte Scheibe fallen normal ebene Lichtwellen auf. Man verändere nun bei gegebener konstanter Winkelgeschwindigkeit so lange die „Phase" der linken Scheibe gegenüber der rechten, bis die linke Scheibe ein Maximum von Licht durchläBt. (Hinter der linken Scheibe sammle eine Konvexlinse das Licht in einen Brennpunkt unabhängig von der Scheibenstellung.) Man erkennt hier sofort die ursprüngliche Übereinstimmung mit dem in der Figur dargestellten Aberrationsschema.

Was für eine Geschwindigheit messen nun alle diese Methoden? - Nach einer Diskussion zwischen einer Gruppe englischer Physiker ${ }^{1}$ ), bei der zunächst noch der Foucaultschen Methode (rotierender Spiegel) eine Sonderstellung eingeräumt worden war, einigte man sich allgemein dahin, daB alle Methoden (auBer der Aberrationsmethode) die „Gruppen"-Geschwindigkeit $U$ messen: das heiBt diejenige Geschwindigkeit, mit der sich die Gebiete gröBter Erregung verschieben, falls man die Übereinanderlagerung zweier unendlich langer Züge von ebenen Sinuswellen mit sehr nahe benachbarten Wellenlängen $\lambda$, $\lambda+\Delta \lambda$ betrachtet. Dabei hängt bekanntlich $U$ mit der Wellengeschwindigkeit $V$ folgendermaßen zusammen:

$$
U=V-\lambda \frac{\Delta V}{\Delta \lambda}
$$

Zugunsten dieser Behauptung lassen sich, scheint es, zurzeit kaum wehr als folgende Bemerkungen anführen:

1. Die abgeschnittenen Wellenzüge, mit denen alle jene Methoden arbeiten, können erzeugt gedacht werden durch Übereinanderlagerung von unendlich vielen, unendlich langen Sinuswellenzügen aller möglichen Wellenlängen mit passend gewählten Phasen und Amplituden. (Darstellung einer beliebig intermittierenden Erregung durch ein Fourierintegral). Die Übereinanderlagerung von wenigstens zwei Wellenzügen sehr

1) Nature 33. 1886. 
benachbarter Wellenlängen $\lambda, \lambda+\Delta \lambda$ mag vielleicht eine Vorstellung ron dem geben, was in jenem komplizierten Fall geschieht.

2. Die faktisch beobachtete Geschwindigkeit, mit der sich eine beliebig begrenzte Störung auf einer Wasserfläche ausbreitet, soll gut mit $U$ übereinstimmen.

3. Die absolute Messung der Lichtgeschwindigkeit in Schwefelkohlenst off (Michels on mit rotierendem Spiegel) kann mit der Brechungsindexmessung durch die Annahme gerade von $U$ als Fortpflanzungsgeschwindigkeit gut in Einklang gebracht werden. ${ }^{1}$ )

4. Für ein Medium, dessen Dispersion von $\lambda=0$ bis $\lambda=\infty$ durch die Gleichung

$$
V(\lambda)=a+b \lambda
$$

dargestellt wird, konnte Schuster streng beweisen, dab jede beliebig begrenzte Erregung in ihm mit der Geschwindigkeit $U$

wandert. ${ }^{2}$ )

$$
U=V-\lambda \frac{d V}{d \lambda}=\alpha
$$

In allen anderen Fällen auBer dem Schuster schen stöBt die strenge Behandlung der Frage, wie sich eine begrenzte Störung ausbreitet, auf vorläufig nicht überwundene Schwierigkeiten, sobald man bis zur quantitativen Behandlung vordringen will. In einigen Fällen (Wasserwellen) gelang es allerdings für die ersten Momente der Ausbreitung das Verhalten in der nächsten Umgebung des ursprünglichen Störungsgebietes zu analysieren; für die vorliegende Frage kommt es ja aber umgekehrt gerade auf das Verhalten in großer Entfernung an. Solange diese Schwierigkeiten nicht überwunden sind, wird man sich also wohl oder übel an die Annahme halten, daB allgemein $U$ die Ausbreitungsgeschwindigkeit begrenzter Störungen darstellt.

Wie sehr aber die Gültigkeitsgrenzen dieser Annahme einer näheren Untersuchung bedürften, zeigen solche Fälle, bei denen die "Gruppen".Geschwindigkeit $U$ größer ist als die Geschwindig-

1) Vgl. z. B. R. Wood, l. c.

2) A. Schuster, Boltzmann-Festschrift p. 569 . 
keit, mit der die Front vorwärts wandert, d. h. jene Fläche, vor welcher das Medium im gegebenen Moment noch sicher exakt rubt.

Einen solchen Fall liefert - wie W. Voigt zeigte ${ }^{1}$ ) die Telegraphistengleichung:

$$
\frac{\partial^{2} \Phi}{\partial t^{2}}=c^{2} \frac{\partial^{2} \Phi}{\partial x^{2}}-\omega \frac{\partial \Phi}{\partial t} .
$$

Hierher gehört ferner - wie M. Lau e ${ }^{2}$ ) nachwies - das Medium, von dem alle Dispersionstheorien handeln, falls man Wellenlängen betrachtet, die in das Gebiet der selektiven $A b$ sorption fallen.

Bei der näheren Analyse des von ihm angegebenen Falles beruft sich Hr. Laue besonders auf den Umstand, daB sich hier die Elementarwellen und also auch die sogenannten „Gruppen“ unter so groBer Absorption fortpflanzen, daB der Begriff Gruppengeschwindigkeit überhaupt keinen physikalischen Sinn besitzt.

Ich möchte deshalb kurz auf die Eigentümlichkeiten hinweisen, die der folgende Fall zeigt.

Es sei eine Saite gegeben, deren unendlich kleine longitudinalen Bewegungen der Gleichung

$$
\frac{\partial^{2} u}{\partial t^{2}}=\alpha^{2} \frac{\partial^{2} u}{\partial x^{2}}+\beta^{2} u
$$

genügen, $d . h$. eine gewöhnliche Saite, bei der aber noch jeder Punkt durch eine Kraft $\beta^{2} u$ aus seiner Ruhelage gedrängt wird.

1. Ruhend und undeformiert ist die Saite im (instabilen) Gleichgewicht.

2. Eine ursprünglich begrenzte Störung breitet sich (mehr und mehr anwachsend) mit der Frontgeschwindigkeit $\alpha$ über die usprünglich ruhende Saite aus. ${ }^{3}$ )

1) W. Voigt, Wied. Ann. 68. p. 598. 1899; Ann. d. Phys. 4. p. 203. 1901 .

2) M. Laue, Ann. d. Phys. 18. p. 523. 1905. \$ 6.

3) Dieses Resultat erhält man, wenn man mit Hilfe der Riemannschen Integrationsmethode (Riemann-Weber, Bd. II. $\$ 121$; W. Voigt, l. c.) die Gleichung (5) bei gegebenen Anfangsbedingungen integriert. 
3. Unendlich lange Sinuswellenzüge wandern ohne Formänderung mit der Wellengeschwindigkeit

$$
V(\lambda)=\alpha\left(1-\lambda^{2} \frac{\beta^{2}}{4 \pi^{2} \alpha^{2}}\right)^{1 / 2} .
$$

4. Die „Gruppen", welche bei der Überlagerung zweier unendlich langer Sinuswellenzüge von den Wellenlängen $\lambda$, $\lambda+d \lambda$ auftreten, wandern mit der Gruppengeschwindigkeit

$$
U(\lambda)=\alpha \frac{1}{\left(1-\lambda^{2} \frac{\beta^{2}}{4 \pi^{2} \alpha^{2}}\right)^{1 / 2}} .
$$

Wie man sieht, ist also für alle Werte von $\lambda$, welche $V$ und $U$ reell machen, die Gruppengeschwindigkeit $U$ größer als die Frontgeschwindigkeit $\alpha$.

Petersburg, Oktober 1910.

(Eingegangen 21. Oktober 1910.) 